\title{
A EXPRESSÃO MORFOFONOLÓGICA DA CONCORDÂNCIA DE NÚMERO VARIÁVEL EM PORTUGUÊS BRASILEIRO: UMA PROPOSTA PARA A AQUISIÇÃO E A PRODUÇÃO INFANTIL
}

\section{THE MORPHOPHONOLOGICAL EXPRESSION OF VARIABLE NUMBER AGREEMENT IN BRAZILIAN PORTUGUESE: AN ACCOUNT OF PRESCHOOLERS' ACQUISITION AND PRODUCTION}

\author{
Ana Paula da Silva Passos Jakubów \\ Universidade do Estado do Rio de Janeiro \\ Rio de Janeiro, Rio de Janeiro, Brasil \\ Letícia M. Sicuro Corrêa \\ Pontifícia Universidade Católica do Rio de Janeiro \\ Rio de Janeiro, Rio de Janeiro, Brasil
}

RESUMO: Crianças brasileiras estão expostas a input de concordância de número variável (NARO, 1981; SCHERRE, 1991; SCHERRE; NARO, 1998). Explora-se, neste artigo, uma possível caracterização da representação e da produção da variação morfofonológica da concordância de número no português brasileiro. Apresentam-se os resultados de um experimento de produção eliciada conduzido com crianças em idade préescolar de escolas pública (EscPub) e particular (EscPriv) no Rio de Janeiro, com vistas a verificar a preferência das crianças por formas redundantes (plural no artigo, no nome e no verbo) e formas não-redundantes (plural apenas no artigo), e variações entre as duas formas. EscPub não apresenta preferência em relação à concordância de número interna ao DP sujeito, enquanto EscPriv prefere a forma redundante do DP. Parte-se de um modelo procedimental da aquisição da linguagem, segundo o qual a criança extrai informação gramaticalmente relevante dos padrões morfofonológicos legíveis na interface fônica (CORRÊA, 2009, 2014). Diante de input variável, sugere-se que a representação gramatical da expressão morfofonológica de número plural estaria subespecificada, sendo, portanto, sujeita a fatores de frequência e informações de uso no momento da codificação morfofonológica na produção da linguagem (ADGER, 2007; LEVELT, 1999; YANG, 2002), o que acarretaria variação. 
PALAVRAS-CHAVE: Variação; Aquisição;

Concordância de número; Psicolinguística; Teoria linguística.

ABSTRACT: Brazilian children are necessarily exposed to variable number agreement (NARO, 1981; SCHERRE, 1991; SCHERRE; NARO, 1998). This article presents a possible characterization of the representation and production of morphophonological variation of number agreement in Brazilian Portuguese by preschoolers from private and public schools in Rio de Janeiro. Preschoolers' preference for standard or non-standard varieties is verified by means of an elicited production task. Results show that public school preschoolers do not present any preference regarding possible varieties of morphophonological number agreement, whereas private school preschoolers prefer the standard form, in which number is redundantly marked in the morphology. It is argued that the processing of variable input in the phonetic/phonological interface results in underspecified representation of morphophonological information in the lexicon (CORREAA, 2009, 2014). The underspecification of morphophonological features in the lexicon would be subject to frequency factors, as well as use information in the moment of morphophonological encoding in language production (ADGER, 2007; LEVELT, 1999; YANG, 2002).

KEYWORDS: Variation; Acquisition; Number agreement; Psycholinguistics; Linguistic theory. 


\section{INTRODUÇÃO}

A variação da concordância de número no Português Brasileiro $(\mathrm{PB})$ é um tópico amplamente explorado pelos estudos sociolinguísticos variacionistas (ALMEIDA, 2010; BRANDÃO, 2015; BRANDÃO; VIEIRA, 2012; NARO, 1981; NARO; SCHERRE, 2015; SCHERRE, 1978，1991，1994; SCHERRE; NARO, 1998; VIEIRA; BRANDÃO, 2014 entre muitos outros). A produção variável da concordância de número está relacionada ao grau de escolaridade do falante, intimamente relacionado com nível socioeconômico (NSE) na realidade brasileira (ver NARO, 1981 e relatório PNAD 2014 do IBGE) ${ }^{1}$. Quanto maior o nível de escolaridade do falante, maior a produção da concordância de plural redundante nos enunciados:

(1) Concordância verbo/sujeito:

... Esses cara hoje só quer curtir mesmo, né?... (SCHERRE; NARO, 1998)

... As dúvidas faz parte...

... As pessoas não toma atitude

...As atitudes ruins afeta... (ALMEIDA, 2010)

(2) Concordância entre os elementos do sintagma nominal:

... essas estradas nova...

... do meus pais...

(3) Concordância nos predicativos e particípios passivos:

... as coisas tão muito caras, né? ...

... que as coisa 'tá cara, num dá mesmo... (SCHERRE; NARO, 1998)

Este artigo baseia-se na sólida constatação da sociolinguística de que a produção da concordância de número em $\mathrm{PB}$ é variável e visa caracterizar a representação e a produção da expressão morfofonológica de número plural por crianças falantes de PB em idade pré-escolar.

O estudo da variação tem sido pouco explorado no âmbito da Psicolinguística, principalmente no que se refere à aquisição da linguagem (DE VOGELAER et al., 2017). Do ponto de vista do processamento, sabe-se, no entanto, que tanto a compreensão quanto a produção de adultos são afetadas por fatores sociais como NSE.

Miller \& Schmitt $(2010,2012)$ contrastam a aquisição da expressão morfofonológica de plural do espanhol mexicano e chileno por crianças e adultos de diferentes NSE. O espanhol mexicano apresenta concordância de número redundante, expressa em todos os elementos da sentença e o espanhol chileno apresenta concordância variável. As autoras verificaram que as crianças chilenas de NSE baixo apresentam maior grau de variação do que as crianças chilenas de

\footnotetext{
${ }^{1}$ Pesquisa Nacional por Amostra de Domicílios 2014 elaborado pelo Instituto Brasileiro de Geografia e Estatística (IBGE). Disponível em:

$<$ https://ww2.ibge.gov.br/home/estatistica/populacao/trabalhoerendimento/pnad2014/def ault.shtm>. Acesso em: 16 dez. 2017.
} 
NSE alto. No processamento adulto, Squires (2014) verifica que fatores sociais como etnia e NSE influenciam o tempo de leitura de estruturas com concordância variável. Falantes de NSE mais baixo e de grupos raciais de minoria tendem a apresentar tempos maiores de leitura, o que, segundo a autora, reflete o baixo desempenho em habilidades de leitura e letramento por esses grupos, ainda que a forma não padrão acarrete tempos de leitura maiores, independentemente de grupo social. No Brasil, estudos sobre o processamento da variação são bastante recentes e vêm mostrando que falantes adultos com alto grau de escolarização produzem a variedade ${ }^{2}$ padrão com maior frequência, mas também aceitam e produzem a variedade não-padrão. $\mathrm{O}$ tempo de leitura para estímulos com concordância não-redundante é maior do que o tempo de leitura para estímulos com concordância redundante (ver AZALIM, 2016; COSTA, 2013; HENRIQUE, 2016; MARCILESE et al., 2015, 2017). No que concerne à aquisição, o grau de variação no input influencia a produção da concordância de número pela criança em idade pré-escolar (MOLINA; MARCILESE; NAME, 2017).

Neste artigo, são apresentados dados experimentais obtidos por meio de tarefa de produção eliciada de modo a caracterizar a representação e a produção da concordância de número variável à luz da teoria linguística minimalista (CHOMSKY, 1995) e de um modelo procedimental de aquisição da linguagem, que incorpora o conceito de interfaces e a hipótese de que toda a informação gramaticalmente relevante para a aquisição de uma língua se encontra legível nas interfaces (fonética/semântica) da língua (interna), com os sistemas de desempenho (CORRÊA, 2009, 2014). Partindo-se da hipótese de que NSE influencia a preferência pelas variedades padrão e não-padrão da concordância de número no momento da produção de enunciados por crianças em idade préescolar, busca-se compatibilizar representação gramatical e sistemas de uso (ADGER, 2007) no momento da codificação morfofonológica (LEVELT, 1989), admitindo-se a interferência de fatores sociais.

Na primeira seção, discute-se a noção de aquisição de uma língua como identificação de traços com base no input recebido. Na segunda seção, apresentam-se as características da concordância variável em PB. Em seguida, o experimento conduzido é apresentado e os resultados são discutidos com vistas a fornecer uma caracterização da produção de número variável em PB. Por fim, discutem-se possíveis contribuições da presente proposta.

\section{A IDENTIFICAÇÃO DA GRAMÁTICA COMO IDENTIFICAÇÃO DE TRAÇOS}

Corrêa $(2009,2014)$ assume a proposta de inicialização da aquisição de uma dada língua por meio de bootstrapping fonológico. A segmentação de enunciados com base na prosódia da língua começaria desde muito cedo. Por

\footnotetext{
${ }^{2}$ Utiliza-se o termo variedade, com base em Labov (1964, 1972, 1997, 2008), o qual estabelece variantes fonéticas de uma dada variável, como os sons possíveis (variantes) para o /-r/, variável, na variedade inglesa. Variedade abrange manifestações linguísticas sociais ou regionais.
} 
volta de 9-12 meses de idade, bebês adquirindo PB reconhecem modificações em afixos morfofonológicos verbais (BAGETTI, 2009; BAGETTI; CORRÊA, 2013). A segmentação de unidades de som no fluxo da fala permitiria à criança a delimitação de constituintes e o estabelecimento de fronteiras de palavras, bem como a identificação da direcionalidade dos núcleos de constituintes. A partir do processamento inicial na interface fônica, as crianças começariam a perceber padrões recorrentes no input, os quais indicariam relevância gramatical da informação codificada nessa interface. Itens recorrentes, sonoramente curtos e em quantidade limitada, seriam identificados mais facilmente dentre o material acústico; estes constituiriam itens de classe fechada, por exemplo, determinantes, preposições, afixos morfológicos nos verbos e nos nomes (SHI, 2014). Itens de classe fechada pertencem às categorias funcionais da língua, as quais projetam informações referentes a propriedades de sistemas intencionais, relacionadas diretamente à intenção da mensagem expressa pelo falante e decodificada pelo ouvinte. A identificação de itens de classe fechada permitiria uma primeira distinção de classes de palavras (itens de classe fechada e outros, que seriam itens de classe aberta). Tal distinção seria representada, em termos de traços, de maneira subespecificada. A presença de traços, ainda que subespecificados morfossintaticamente, deflagraria a operacionalização do sistema computacional. Com maior exposição ao input, os traços seriam paulatinamente adquiridos e especificados, promovendo a identificação dos traços que compõem a gramáticaalvo.

Sendo assim, nessa perspectiva, a aquisição de uma língua se dá via identificação de informação gramatical legível na interface fônica, a ser representada como traços formais de itens lexicais (CORRÊA, 2009, 2014, ver também ADGER, 2006; ADGER \& SMITH, 2010). No Programa Minimalista (PM) (CHOMSKY, 1995), os traços que compõem os itens lexicais podem ser: a) semânticos, os quais representam a codificação do significado de itens lexicais; b) fonológicos, os quais representam as características fônicas relacionadas a morfologia dos itens linguísticos; c) formais - tais como pessoa, número, gênero, tempo, aspecto - operados exclusivamente pelo sistema computacional e alvo de descrição e investigação da teoria linguística formal de base gerativista.

Os traços formais podem ser caracterizados como não-interpretáveis em correspondência com traços interpretáveis, sendo estes últimos lidos pelas interfaces Forma Lógica e Forma Fonética, onde sentido e som são atribuídos a uma estrutura derivada hierarquicamente pelo sistema computacional. $\mathrm{O}$ pareamento entre traços formais interpretáveis e não-interpretáveis são manipulados pelo componente sintático por meio da operação Agree. Essa operação combina os elementos dispostos numa relação de c-comando por meio de valoração (CHOMSKY, 1995; PESETSKY; TORREGO, 2007): um item com um traço $\mathrm{F}$ não-valorado sonda em seu domínio de c-comando um alvo que contenha $\mathrm{F}$ valorado com o qual possa compartilhar seu valor. Dessa forma, Agree pode ser entendida como uma operação estritamente sintática, que pode ter contrapartes para a interpretação semântica do objeto sintático e para a expressão morfofonológica do enunciado. Agree revela para as interfaces a relação estabelecida entre os elementos da estrutura; concordância, portanto, tal como a 
conhecemos tradicionalmente, é a expressão morfofonológica do resultado de Agree, a qual pode estar morfofonologicamente visível nas interfaces ou não (ver MIYAGAWA, 2010). Logo, categorias funcionais podem encontrar na morfo(fono)logia das línguas expressões que identifiquem suas propriedades morfossintáticas; e são as categorias funcionais, itens de classe fechada, as primeiras a serem identificadas pela criança (CORRÊA, 2009; SHI, 2014). Neste artigo, verifica-se a expressão morfofonológica de número presente nas categorias funcionais expressas no sujeito $\mathrm{DP}^{3}$ e no verbo, o qual se move para o núcleo de TP em PB.

Dado que o input de concordância de número plural é variável em PB e, combinando os pressupostos da teoria linguística minimalista com os pressupostos de um modelo procedimental de aquisição, tem-se que a variação pode ser representada na gramática em termos de subespecificação de traços no léxico. Esta proposta é avançada por Adger \& Smith (2010), os quais propõem que a variação intra-indivíduo - oscilação em um mesmo indíviduo ou presença de variantes em uma mesma variedade - emerge da subespecificação no mapeamento entre formas fonológicas (ou morfológicas) e traços morfossintáticos (ibid, p. 1112). Compartilha-se esta ideia e assume-se a perspectiva de Corrêa $(2009,2014)$ acrescentando que a subespecificação no mapeamento da realização morfofonológica de categorias funcionais pode ser representada no léxico como subespecificação de informações morfofonológicas para a expressão de plural em PB.

Entende-se subspecificação como a representação de um traço que, sendo uma variável, poderia assumir diferentes valores ou manter-se sem valor definido (ver ROORYCK, 1994 para dois tipos de subespecificação). ${ }^{4}$ Em suma, subespecificação de traços pertinentes à informação morfofonológica seria uma consequência direta da exposição a input variável. ${ }^{5}$ A extração de informações

\footnotetext{
${ }^{3}$ Utiliza-se, conforme a literatura gerativista minimalista, a nomenclatura DP para se referir ao sujeito composto por um determinante e um nome e TP para designar a relação Spec-head estabelecida entre sujeito e verbo quando o DP é movido para Spec,TP, onde o traço [número] do verbo pode, então, ser checado de acordo com o DP sujeito.

${ }^{4}$ Subespecificação também é um conceito adotado pela Morfologia Distribuída (MD) (EMBICK, 2015; HALLE; MARANTZ, 1993; NEVINS; PARROTT, 2010). No entanto, neste artigo, assume-se a visão de Rooryck (1994): "At first sight, underspecified $\alpha$-valued features simply appear to be unspecified features, but I argue that their complete absence of specification plays a role in the grammar 'variable' or $\alpha$ features have no 'fixed' value, but can 'pass on' the features of the elements surrounding them" (ROORYCK, 1994, p. 209 - destaques do autor).

${ }^{5}$ Costa \& Figueiredo Silva (2006) propõem com base na Morfologia Distribuída (EMBICK; NOYER, 2001) que o morfema de número é representado de forma diferenciada em PB e em PE. Enquanto no PB, este seria um singleton, presente na sintaxe, relevante para a interpretação semântica e ancorado no determinante (ou em elementos pré-nominais), em PE, este seria dissociado, ou seja, as formas redundantes seriam inseridas pós-sintaticamente e não seriam relevantes para a interpretação semântica. Os autores consideram ainda que a configuração Spec-head seria um desencadeador da expressão da concordância sujeito-verbo de número, ainda que não
} 
gramaticais a partir do fluxo da fala forneceria dois possíveis valores para plural em PB: $/-\mathrm{s} /{ }^{6}$ ou $/-\varnothing /$. Aventa-se a hipótese de que a inserção de um ou outro morfema estaria sujeita a fatores externos sociais e de uso, os quais alterariam o peso de cada variedade representada no léxico (YANG, 2002).

Dessa forma, a hipótese desta investigação é que, dada a exposição a input variável, a expressão morfofonológica de número seria representada como subespecificada no nome - que compõe o sujeito da sentença, projetado sob a categoria funcional DP - e no verbo - o qual concorda com o sujeito, que, em PB, sobe para o núcleo $\mathrm{T}$ para checar os traços de tempo e modo. Sendo assim, a expressão morfofonológica de número plural em $\mathrm{PB}$ é obrigatória apenas em D (AUGUSTO; FERRARI NETO; CORRÊA, 2006; FERRARI NETO, 2008; LOPES, 2004, 2006; MAGALHÃES, 2004). Assume-se, assim, que a codificação de número gramatical, distinção entre singular e plural, se dá no núcleo da categoria funcional Num em NumP (Number Phrase) (RITTER, 1995). Num está localizado abaixo de D no DP e a manifestação do valor de Num em D é produto de Agree no DP. Assim, o que é subespecificado com relação à expressão morfonofológica de número diz respeito à redundância na morfologia.

\section{CARACTERÍSTICAS DA CONCORDÂNCIA DE NÚMERO VARIÁVEL EM PB}

O grau de variação na expressão morfofofonológica de número na produção de adultos é condicionado a fatores linguísticos e extra-linguísticos, como evidenciado na literatura sociolinguística. No que concerne aos fatores linguísticos, saliência fônica e marcação de plural em elementos à esquerda do núcleo, dentre outras propriedades como paralelismo, têm sido verificadas (BRANDÃO, 2013; SCHERRE; NARO, 1998). Em relação aos fatores extra-

obrigatoriamente, tanto em PE, quanto no que denominam a variedade PB1, que apresenta concordância de número no verbo, independentemente de como esta é expressa no DP, em contraste com a variedade PB2, em que o número é expresso somente no determinante. A ideia de que há diferenças entre o tipo de morfema no PE e no $\mathrm{PB}$, nos termos da Morfologia Distribuída, pode, em princípio, ser sustentada à luz de dados sociolinguísticos. Contudo, tal distinção não contempla, por um lado, a existência de uma variedade em PB semelhante ao PE (variedade padrão) e, por outro, traz uma distinção entre PB1 e PB2 pouco sustentável. Não há na literatura sociolinguística evidência para ocorrências sistemáticas de uma ou outra forma que permitam distinguir grupos de falantes por seu uso. De fato, o que se observa é variação intra-indivíduo ("eles ganha demais (...)" / "eles ganham demais da conta" (Scherre \& Naro, 1998), o que torna pouco provável que seja a configuração Spec-head o que deflagre a ocorrência de uma ou outra forma. Assim sendo, a distinção entre variedades nos termos propostos por Costa \& Figueiredo Silva não contempla a grande flutuação que se observa na presença de marcas redundantes em variedades não-padrão do PB (ver também SILVA; MAGALHÃES, 2014).

${ }^{6}$ Apesar de não ser o morfema de plural utilizado para verbos, a notação /-s/ é utilizada de maneira genérica para morfema de plural de modo a garantir simplicidade de informação ao longo da apresentação do texto. 
linguísticos, o nível de escolaridade do falante é a característica mais proeminente na produção da concordância de número variável, configurando um fator social. A variedade não-padrão, a que não expressa marcação de número plural redundante, está, portanto, sujeita ao estigma social, relacionado à falta de escolaridade do falante (BAGNO, 2007). Vieira (2015) apresenta o nível de variação na produção da concordância de número sujeito-verbo de acordo com o nível de escolaridade do falante na população do Rio de Janeiro:

Tabela 1: Continuum para a marcação de plural em concordância de número em verbos de $3^{\mathrm{a}}$ pessoa do plural em PB de acordo com nível de escolaridade

\begin{tabular}{|c|c|c|}
\hline \multicolumn{3}{|c|}{+ marcas de plural $\longleftrightarrow$ - marcas de plura } \\
\hline \multicolumn{3}{|c|}{ Área urbana do Rio de Janeiro } \\
\hline Nível de escolaridade alto & Nível de escolaridade Médio & Não-letrado \\
\hline $\begin{array}{c}\mathbf{9 4 \%} \\
(\text { Graciosa,1991) } \\
\mathbf{9 7 , 8 \%} \\
\text { (Vieira \& Bazenga, 2013) }\end{array}$ & $\begin{array}{c}\mathbf{7 3 \%} \\
\text { (Scherre \& Naro,2006) } \\
\mathbf{8 9 \%} \\
\text { (Vieira \& Bazenga, 2013) }\end{array}$ & $\begin{array}{c}\mathbf{4 8 \%} \\
\text { (Naro,1981) }\end{array}$ \\
\hline
\end{tabular}

Fonte: (adaptado de VIEIRA, 2015, p. 114 - Figura 2)

A dinâmica da realidade social brasileira, principalmente em grandes centros urbanos, permite a formação de teias de contato entre pessoas de diferentes $\mathrm{NSE}^{7}$, o que também promove a difusão de características da língua, como a concordância de número em PB (MILROY; MILROY, 1985). É nesse contexto de contato entre diferentes variedades linguísticas, especificamente, no que concerne à concordância de número variável que a criança deve identificar, processar, representar e utilizar as possíveis variedades às quais está exposta desde o nascimento (ver MUFWENE, 2001, 2008, 2011 para discussões acerca da ecologia da língua e um modelo para línguas em contato).

Na próxima seção, apresenta-se um experimento de produção induzida por repetição com vistas a verificar preferências pelas variedades padrão e não-padrão da expressão morfofonológica da concordância de número em PB na produção de crianças em idade pré-escolar no subúrbio do Rio de Janeiro.

\footnotetext{
${ }^{7}$ A Associação Brasileira de Empresas de Pesquisa tem adotado o Critério Brasil com vistas a classificar a população de acordo com o poder de compra ou rendimento médio, associados ao grau de instrução do chefe de família, sugerindo uma classificação econômica. Disponível em: $<$ http://www.abep.org/criterio-brasil $>$

Acesso em 18 dez. 2017. Ver, também, suplemento de Mobilidade Sócio-ocupacional do último relatório do PNAD do IBGE (2014). Disponível em: $<$ https://agenciadenoticias.ibge.gov.br/media/com mediaibge/arquivos/331e3fd38ba3dc e6411dfe876b4c0f76.pdf> Acesso em 18 dez. 2017.
} 


\section{A PRODUÇÃO DA CONCORDÂNCIA DE NÚMERO EM PB EM FASE PRÉ- ESCOLAR}

Dados na literatura psicolinguística demonstram, via metodologia experimental, que, aos 2 anos de idade, a criança adquirindo PB é sensível às propriedades de número expressas apenas em D (FERRARI NETO, 2008; FERRARI NETO; CORREAA; AUGUSTO, 2005). No presente estudo, foi proposto um experimento de produção eliciada por repetição com vistas a verificar:

1) se há preferência por uma ou mais formas de expressão morfofonológica de número gramatical em crianças adquirindo o PB;

2) se a variável social definida pelo tipo de escola (privada ou pública) afeta a preferência por uma dada forma de expressão, assumindo que o tipo de escola que as crianças frequentam reflete propriedades do NSE do falante (ALVES; SOARES; XAVIER, 2014).

\subsection{Design do experimento}

Foram manipuladas as seguintes variáveis independentes: 1. Redundância no DP (redundante; não-redundante); 2. Redundância no TP (redundante, nãoredundante) e 3. Tipo de escola (pública; privada), totalizando 4 condições experimentais:

Tabela 2: Condições experimentais do experimento de produção eliciada por repetição

\begin{tabular}{|l|l|}
\hline Condição 1 (DP red/ TP red) & Os cachorros encontraram o leão \\
\hline Condição 2 (DP red/TP não-red) & Os cachorros encontrou o leão \\
\hline Condição 3 (DP não-red/ TP red) & Os cachorro encontraram o leão \\
\hline Condição 4 (DP não-red/ TP não red) & Os cachorro encontrou o leão \\
\hline
\end{tabular}

Fonte: As autoras

A expressão morfofonológica de número plural foi mantida em $\mathrm{D}$ em todos os estímulos, já que número gramatical é semanticamente interpretável em D. Diante das quatro possibilidades de combinação, sendo uma condição padrão (condição 1), e outras combinações não-padrão (condições 2, 3 e 4), acredita-se que a criança reproduz o estímulo quando este estiver de acordo com sua gramática ou o corrige, acrescentando ou retirando marcas de plural, em função daquilo que sua gramática é capaz de gerar (SLOBIN; WELSH, 1967). A variável dependente foi o número de respostas que reproduziram as expressões morfofonológicas de número do estímulo. 


\subsection{Método}

\subsubsection{Participantes}

Foram analisados os dados de 20 crianças de uma escola privada no subúrbio do Rio de Janeiro (grupo: EscPriv; 5;0 a 5;11 anos; média de idade: 5;7 anos), sendo 8 meninas e 12 meninos, e 16 crianças de uma escola pública do mesmo bairro (grupo: EscPub; 5;0 a 6;3 anos; média de idade: 5;9 anos), sendo 6 meninas e 10 meninos.

\subsubsection{Material}

Foram criados 4 estímulos, com 8 - 11 sílabas, para cada uma das 4 condições, totalizando 16 estímulos experimentais. O tipo de verbo - transitivo de ação - e a animacidade e o gênero do complemento do verbo foram controlados. Cada criança foi exposta a estímulos de todas as condições. Foram elaboradas 4 listas com 16 estímulos semi-aleatorizados de acordo com o princípio do quadrado latino; não foram apresentados estímulos de uma mesma condição consecutivamente.

Os estímulos foram gravados e editados no software Audacity para alteração de pitch de modo a soar mais amigável para a criança. Os estímulos foram reproduzidos pelo $B o b$, um boneco animado no computador via software, Crazy Talk 2 da Reallusion. As respostas das crianças foram registradas por gravadores de voz Sony MP3 player-recorder ou Panasonic RR-US511. O experimento foi realizado em um laptop DELL Inspiron com auxílio de caixas de som.

\subsubsection{Procedimento}

Utilizou-se a técnica de produção induzida por repetição. As crianças ouviram o seguinte protocolo antes de serem expostas aos estímulos: $O$ Bob mora em um lugar muito longe e ele fala um pouco diferente da gente. Lá onde ele mora, acontece um monte de coisas. Ele vai te contar o que aconteceu, mas eu não vou ouvir. O Bob disse que você tem que ouvir e me contar, tá bom? Então, eu vou tampar os ouvidos e você me conta o que aconteceu lá onde o Bob mora. As crianças realizaram a tarefa individualmente em salas disponibilizadas pelas escolas e as respostas foram gravadas, transcritas e analisadas de acordo com a correspondência às expressões morfofonológicas de número presentes nos estímulos. Respostas que corrigiam o estímulo também foram registradas e analisadas.

\subsection{Resultados}

O gráfico abaixo apresenta o escore médio de cada grupo em relação às respostas correspondentes ao estímulo fornecido: 
Gráfico 1: Médias por condição por tipo de escola; escore máximo = 4; barras de erro: desvio-padrão

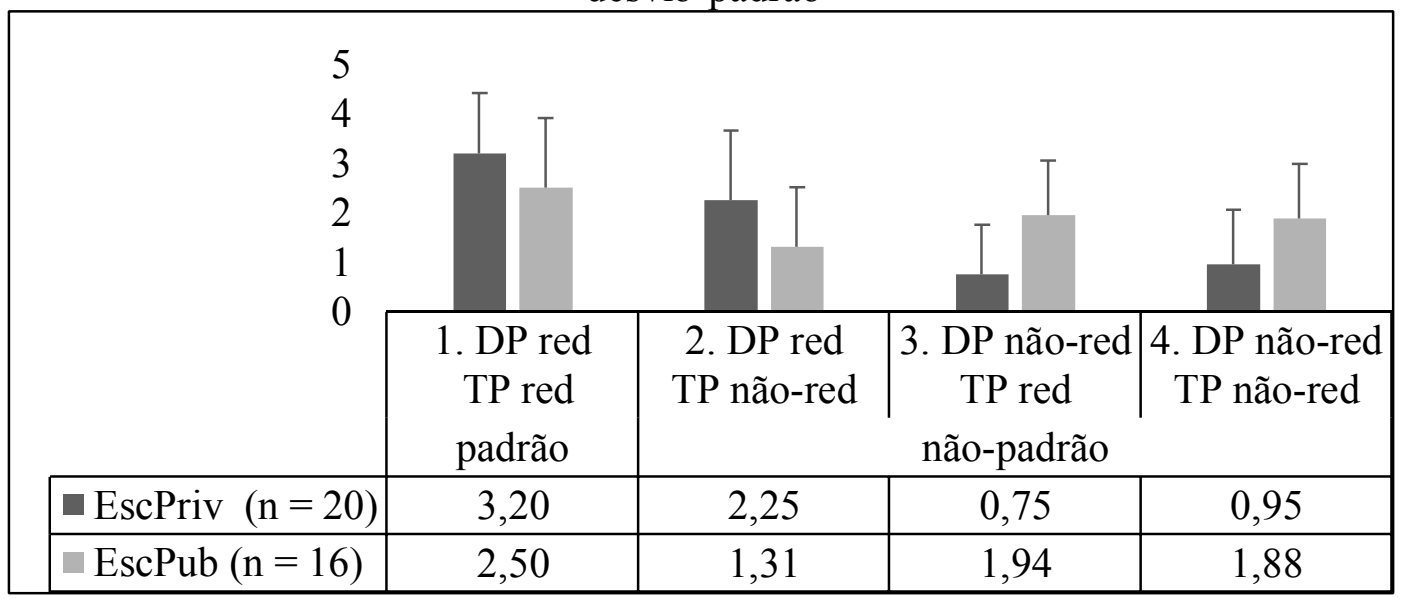

Fonte: As autoras

Um teste de amostras independentes, Mann-Whitney $U$, revelou diferença entre os grupos nas condições: $2(U=97.5 ; p=.046), 3(U=68 ; p=.003)$ e 4 ( $U$ $=86 ; p=.018)$. Na condição 1 , variedade padrão, não foi verificada diferença entre os grupos $(U=109.5 ; p=.109)$. Sendo assim, ambos os grupos apresentam preferência pela concordância de número redundante, porém o grupo EscPub aceita a variante não-redundante com mais frequência do que o grupo EscPriv. Ao analisar as respostas em termos de redundância dentro de um mesmo constituinte, DP ou TP, um resultado interessante é constatado:

Gráfico 2: Média de respostas por redundância no DP e no TP por tipo de escola; escore máximo $=8$; barras de erro desvio-padrão

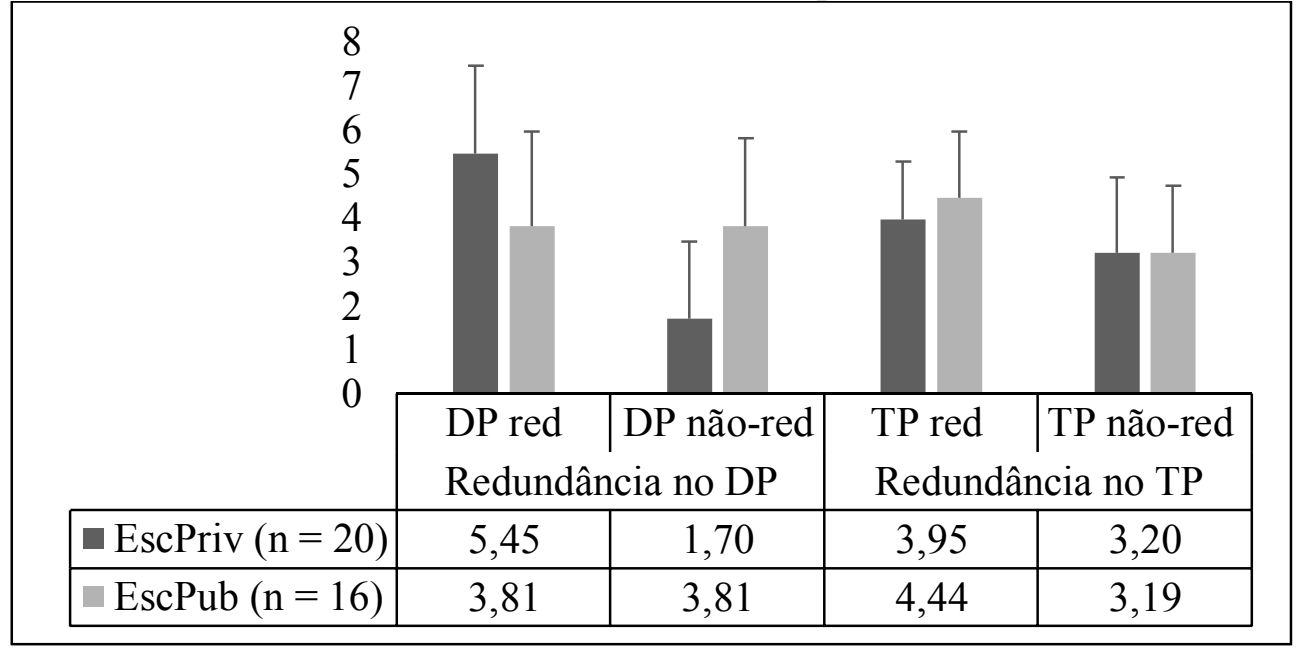

Fonte: As autoras

Um teste de amostras independentes, Mann-Whitney $U$, revelou efeito significativo para redundância no DP entre grupos (DP red: $U=91 ; p=.028 / \mathrm{DP}$ não-red: $U=68,5 ; p=.003)$, mas não foi verificado efeito significativo entre grupos em relação à redundância no $\mathrm{TP}(\mathrm{TP}$ red: $U=113 ; p=.140$ / TP não-red: $U=159.5 ; p=.987)$. O grupo EscPub não apresentou preferência em relação à redundância no DP e o grupo EscPriv apresentou preferência por DP redundante. 
Ao analisar os tipos de respostas para cada condição, verifica-se que o grupo EscPriv tende a reproduzir o estímulo ou a corrigir os estímulos para a concordância redundante, principalmente na condição 3 (DP não-red/ TP red) e a condição 4 é a que tem o desempenho mais variado:

Tabela 2: Distribuição (\%) de respostas por cada condição no grupo da Escola Privada (*Total de respostas válidas)

\begin{tabular}{|c|c|c|c|c|c|c|c|c|c|}
\hline \multirow[b]{2}{*}{$\begin{array}{l}\text { Condições } \\
\text { apresentadas }\end{array}$} & \multicolumn{8}{|c|}{ Tipos de respostas por condição - EscPriv $(n=20)$} & \multirow[b]{2}{*}{ Total } \\
\hline & 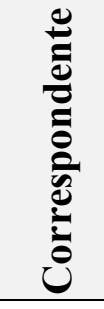 & 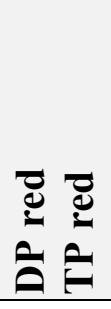 & 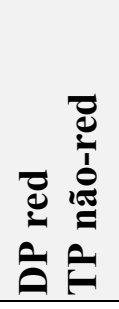 & 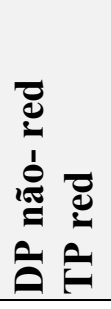 & 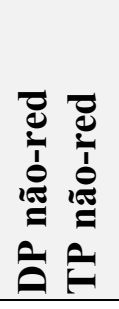 & 苛 & ڤે & 苞 & \\
\hline $\begin{array}{r}\text { C1: } \text { DP red } \\
\text { TP red }\end{array}$ & $80 \%$ & $0 \%$ & $0 \%$ & $8 \%$ & $3 \%$ & $3 \%$ & $3 \%$ & $5 \%$ & $\begin{array}{c}80 * \\
(100 \%) \\
\end{array}$ \\
\hline $\begin{array}{l}\text { C2: DP red } \\
\text { TP não-red }\end{array}$ & $56 \%$ & $19 \%$ & $0 \%$ & $0 \%$ & $13 \%$ & $11 \%$ & $1 \%$ & $0 \%$ & $\begin{array}{c}80 * \\
(100 \%)\end{array}$ \\
\hline $\begin{array}{c}\text { C3: DP não-red } \\
\text { TP red }\end{array}$ & $19 \%$ & $64 \%$ & $0 \%$ & $0 \%$ & $6 \%$ & $0 \%$ & $3 \%$ & $9 \%$ & $\begin{array}{c}80 * \\
(100 \%) \\
\end{array}$ \\
\hline $\begin{array}{r}\text { C4: DP não-red } \\
\text { TP não-red }\end{array}$ & $24 \%$ & $15 \%$ & $29 \%$ & $3 \%$ & $0 \%$ & $25 \%$ & $3 \%$ & $3 \%$ & $\begin{array}{c}80^{*} \\
(100 \%)\end{array}$ \\
\hline
\end{tabular}

Fonte: As autoras

As correções feitas pelo grupo EscPub demonstram maior grau de flutuação nos tipos de respostas fornecidas em cada condição:

Tabela 3: Distribuição (\%) de respostas por cada condição no grupo da Escola Pública (*Total de respostas válidas)

\begin{tabular}{|c|c|c|c|c|c|c|c|c|c|}
\hline \multirow[b]{2}{*}{$\begin{array}{c}\text { Condições } \\
\text { apresentadas }\end{array}$} & \multicolumn{8}{|c|}{ Tipos de respostas por condição - EscPub $(n=16)$} & \multirow[b]{2}{*}{ Total } \\
\hline & 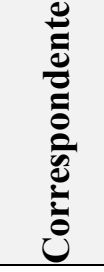 & 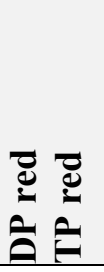 & 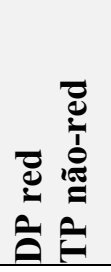 & 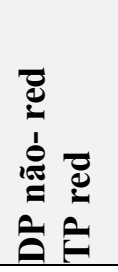 & 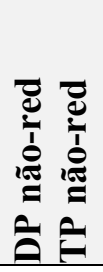 & 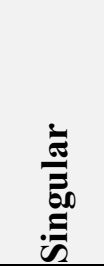 & $\begin{array}{l}\text { Q } \\
\text { E } \\
\text { है }\end{array}$ & 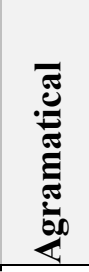 & \\
\hline $\begin{array}{r}\text { C1: DP red } \\
\text { TP red }\end{array}$ & $63 \%$ & $0 \%$ & $0 \%$ & $22 \%$ & $5 \%$ & $0 \%$ & $3 \%$ & $8 \%$ & $\begin{array}{c}64 * \\
(100 \%) \\
\end{array}$ \\
\hline $\begin{array}{l}\text { C2: DP red } \\
\text { TP não-red }\end{array}$ & $33 \%$ & $14 \%$ & $0 \%$ & $3 \%$ & $28 \%$ & $19 \%$ & $0 \%$ & $3 \%$ & $\begin{array}{c}64 * \\
(100 \%)\end{array}$ \\
\hline $\begin{array}{c}\text { C3: DP não-red } \\
\text { TP red }\end{array}$ & $48 \%$ & $38 \%$ & $2 \%$ & $0 \%$ & $2 \%$ & $0 \%$ & $3 \%$ & $8 \%$ & $\begin{array}{c}64 * \\
(100 \%)\end{array}$ \\
\hline $\begin{array}{c}\text { C4: DP não-red } \\
\text { TP não-red }\end{array}$ & $47 \%$ & $3 \%$ & $28 \%$ & $3 \%$ & $0 \%$ & $19 \%$ & $0 \%$ & $0 \%$ & $\begin{array}{c}64 * \\
(100 \%)\end{array}$ \\
\hline
\end{tabular}

Fonte: As autoras 
No momento da participação na atividade, essas crianças ainda não sabiam ler ou escrever e não haviam sido formalmente apresentadas à norma padrão escrita do PB. Sendo assim, esses dados parecem refletir a gramática adquirida naturalmente pela criança. Verifica-se, portanto que, considerando o tipo de escola como variável social, que caracteriza o NSE do falante, há graus distintos de variação na produção de concordância entre os grupos de pré-escolares. O grau de variação é interpretado como subespecificação na representação da expressão morfofonológica pertinente a marca de plural no PB. Essa interpretação é discutida na próxima seção. ${ }^{8}$

\section{VARIAÇÃO COMO SUBESPECIFICAÇÃO DA EXPRESSÃO MORFOFONOLÓGICA DE NÚMERO}

Nesse artigo, avançou-se a hipótese de que input variável acarreta representação da expressão morfofonológica da concordância subespecificada no léxico. A teoria linguística apresenta um recurso simples, porém poderoso, para dar conta de propriedades variáveis: a subespecificação (ROORYCK, 1994). Uma representação de traço que não assuma nem um valor, nem outro diante de input variável, parece ser uma consequência plausível de exposição à variação. Se a expressão morfofonológica de número é representada como subespecificada, a variação da concordância de número no $\mathrm{PB}$, de fato, emerge em um momento pós-sintático. No componente sintático, traços formais de número dos elementos lexicais são combinados via Agree de forma mecânica e automática. Assume-se, portanto, a sintaxe como um componente imune à variação (BOECKX, 2011; BOECKX; LEIVADA, 2014). Sendo assim, do ponto de vista do processamento, a variação na expressão da concordância de número plural ocorre no momento da codificação morfofonológica durante a produção do enunciado.

Os resultados experimentais obtidos evidenciam a flutuação na produção de marcas de concordância de número plural por crianças em idade pré-escolar, corroborando o estatuto da concordância de número no $\mathrm{PB}$ como regra variável (VIEIRA; BRANDÃO, 2014). Na perspectiva da produção da linguagem, seria no momento do acesso ao léxico, na busca de morfemas para a expressão de concordância de número plural durante a codificação morfofonológica que

\footnotetext{
${ }^{8}$ Um parecerista anônimo mencionou a possibilidade de a saliência fônica da marca de plural no verbo ser relevante para a ocorrência de marcas morfológicas redundantes. A noção de saliência fônica é, contudo, pouco clara (ver CHAVES, 2014). A escala de saliência fônica apresentada em SCHERRE \& NARO (1998) baseia-se fundamentalmente em dois critérios (1) acento tônico e (2) quantidade de material fônico que diferencia a forma singular da forma plural. Não é definido, pelos autores, qual dos critérios teria prioridade sobre o outro. Assim sendo, a única distinção possível quanto à saliência fônica, seria entre + tônico/ + material fônico (como em cantarão, em relação a cantou (+tônico/-saliente) ou em relação a cantaram (- tônico/ + material fônico)). Não haveria, segundo nossa leitura da aplicação desses critérios, previsão de diferença quanto à saliência fônica entre as formas cantou e cantaram presentes no experimento.
} 
informações de frequência (LEVELT, 1999) e uso das variedades, armazenadas numa espécie de Pool of Variants (ADGER, 2007) poderiam atuar. Cada variedade teria um peso distinto conforme as características de uso que as definem na representação gramatical do falante (ver YANG, 2002). O peso atribuído a cada uma das variedades influenciaria o acesso aos morfemas, equivalente ao nível do lexema em modelos psicolinguísticos de produção (cf. ROELOFS; MEYER; LEVELT, 1998; ROELOFS, 1998). Para tanto, assume-se que as expressões morfofonológicas /-s/ ou /-Ø/ seriam armazenadas independentemente no léxico (SCHILLER; COSTA, 2006; STEMBERGER; MACWHINNEY, 1986; TAFT; FORSTER, 1975).

Figura 1: Variação na produção da concordância de número plural em PB

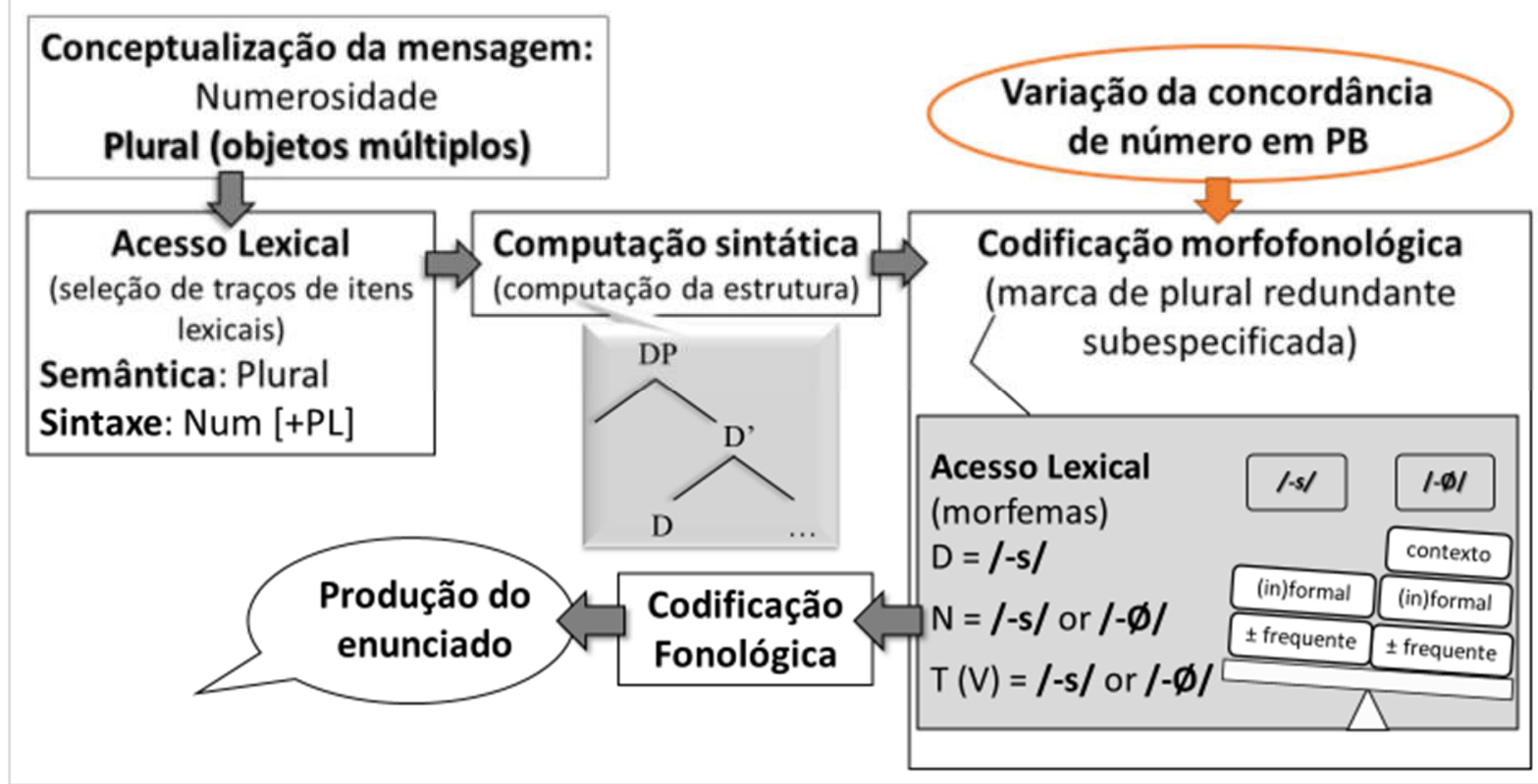

Fonte: As autoras

Portanto, na produção de um enunciado, número plural seria estabelecido na conceptualização da mensagem (LEVELT, 1989), indicando a necessidade de recuperação da categoria Num com valor plural. No componente sintático, ocorreria a derivação da estrutura, onde traços formais dos itens lexicais seriam pareados, sendo os não-interpretáveis valorados, via Agree, o que acarretaria a codificação da expressão morfofonológica de plural em D e interpretação semântica de 'mais de um' nesse item. No momento da codificação morfofonológica, informações de efeitos de uso seriam acessadas e a variedade com maior peso seria inserida. Na codificação fonológica cada item receberia propriedades acústicas, fonéticas e fonológicas, para, então, serem articulados em um enunciado.

Uma série de fatores externos pode contribuir para o status de cada variedade linguística representada pelo falante. Uma delas é NSE que, como visto nos dados experimentais, interfere na representação e no uso dos morfemas de número em PB. Perkins et al. (2013) argumentam que NSE pode influenciar o desenvolvimento cerebral, cognitivo e linguístico da criança. No entanto, não há estudos, até o presente momento, sobre como esses fatores podem influenciar o 
uso de uma característica que parece ser tão específica da língua como o morfema de número. Pace et al. (2017, p. 286) destacam que estudos interdisciplinares de abordagem bioecológica e psicolinguística são escassos, mas salientam que a integração entre as duas áreas poderia contribuir para entendimento mais amplo da relação entre ambientes linguísticos e desenvolvimento linguístico.

\section{CONSIDERAÇÕES}

Neste artigo, foi proposta uma caracterização da representação gramatical e da produção da expressão morfofonológica da concordância de número plural em PB. A exposição a input variável devido ao contato entre variedades linguísticas caracterizadas por fatores sociais acarreta subespecificação de informações pertinentes a traços morfofonológicos no léxico. Para isso, assumiuse o armazenamento de variedades linguísticas em uma espécie de Pool of variants (ADGER, 2007) de forma separada, com informações sobre o uso de cada uma das variedades (YANG, 2002). Verificou-se, por meio de metodologia experimental, que o NSE do ambiente da criança influencia o uso variável da concordância de número plural em $\mathrm{PB}$, mesmo antes da exposição à norma padrão escrita na escola. A exposição à norma padrão durante o ensino formal pode alterar o status das variedades acessíveis ao falante, fazendo com que este atribua maior peso a uma ou outra, dependendo do contexto em que se encontre (LABOV, 2008 [1972]).

Acredita-se que uma caracterização da variação que integre teoria linguística, dados experimentais e modelos de processamento permite explicar a opcionalidade no uso de marcas morfológicas redundantes da concordância de número na produção dos falantes de $\mathrm{PB}$. A subespecificação, antes um recurso reservado a traços formais, no que concerne à sintaxe, quando estendida à informação relevante para a codificação morfofonológica, encontra espaço em um modelo de processamento que explicite como informação gramatical representada no léxico é recuperada em diferentes momentos da produção da fala - na condição gramatical (processamento sintático) e na codificação morfofonológica.

\section{REFERÊNCIAS}

ADGER, D. Combinatorial Variability. Journal of Linguistics, v. 42, n. 03, p. 503, 2006.

ADGER, D. Variability and modularity: A response to Hudson. Journal of Linguistics, v. 43, n. 43, p. 695-700, 2007.

ADGER, D.; SMITH, J. Variation in agreement: A lexical feature-based approach. Lingua, v. 120, n. 5, p. 1109-1134, 2010. 
ALMEIDA, E. M. Uso e norma: variação da concordância verbal em redações escolares. Tese de Doutorado. 206f. Universidade Federal do Rio de Janeiro, 2010.

ALVES, M. T. G.; SOARES, J. F.; XAVIER, F. P. Índice Socioeconômico das Escolas de Educação Básica Brasileiras. Ensaio: aval. pol. públ. Educ., v. 22, n. 84, p. 671-704, 2014.

AUGUSTO, M. R. A.; FERRARI NETO, J.; CORREAA, L. M. S. Explorando o DP : a presença da categoria NumP. Revista de Estudos Linguísticos, v. 14, n. 2 , p. 245-275, 2006.

AZALIM, C. Variação e processamento linguístico: um estudo experimental sobre a concordância nominal variável no PB. Dissertação de Mestrado. 129f. Universidade Federal de Juiz de Fora, 2016.

BAGETTI, T. Um Estudo Experimental do Processamento na Interface Fônica e da Análise Sintática Inicial: O Papel de Elementos Funcionais na Aquisição da Linguagem. Tese de Doutorado. 151f. Pontíficia Universidade Católica do Rio de Janeiro, 2009.

BAGETTI, T.; CORRÊA, L. M. S. O reconhecimento do padrão morfofonológico dos afixos verbais na aquisição inicial do português brasileiro. Veredas atemática, v. 17, n. 2 , p. $102-122,2013$.

BAGNO, M. Preconceito Linguístico: o que é, como se faz. $49^{\mathrm{a}}$ ed. São Paulo: Edições Loyola, 2007.

BOECKX, C. Approaching Parameters from Below. In: BOECKX, C.; DI SCIULLO, A.-M. (Eds.). The Biolinguistic Enterprise: New Perspectives on the Evolution and Nature of the Human Language Faculty. Oxford, UK: OUP Oxford, 2011. p. 205-221.

BOECKX, C.; LEIVADA, E. On the particulars of Universal Grammar: Implications for acquisition. Language Sciences, v. 46, n. PB, 2014.

BRANDÃO, S. F. Patterns of plural agreement within the Noun Phrase. Journal of Portuguese Linguistics, v. 12, n. 2, p. 51-100, 2013.

BRANDÃO, S. F. Concordância nominal em três variedades do português: resultados gerais, novas indagações. Cuadernos de la ALFAL. 2015.

BRANDÃO, S. F.; VIEIRA, S. R. Concordância nominal e verbal: contribuições para o debate sobre o estatuto da variação em três variedades urbanas do português. Alfa, v. 56, n. 3, p. 1035-1064, 2012.

CHAVES, R. G. Princípio de saliência fônica: isso não soa bem. Letrônica, v. 7, n. 2, p. 522-550, 2014. 
CHOMSKY, N. The Minimalist Program. Cambridge, Massachusetts: The MIT Press, 1995.

CORRÊA, L. M. S. Bootstrapping language acquisition from a minimalist standpoint: On the identification of $\varphi$-features in Brazilian Portuguese. Minimalist Inquiries Into Child and Adult Language Acquisition: Case Studies Across Portuguese, v. 35, p. 35-62, 2009.

CORREA, L. M. S. Interface Information and Computational Cost: An Integrated Procedural Approach to Language Acquisition with Some Implications for SLI. New Directions in the Acquisition of Romance Languages, 2014.

CORRÊA, L. M. S.; AUGUSTO, M. R. A. Computação Linguística no processamento on-line: soluções formais para a incorporação de uma derivação minimalista em modelos de processamento. Cadernos de Estudos Lingüísticos, v. 49, n. 2, p. 167-183, 2007.

CORRÊA, L. M. S.; AUGUSTO, M. R. A. Possible loci of SLI from a both linguistic and psycholinguistic perspective. Lingua, v. 121, n. 3, p. 476-486, 2011.

COSTA, I. DE O. Verbos meteorológicos no plural em orações relativas do português brasileiro: sintaxe e processamento. Dissertação de Mestrado. 196f. Pontíficia Universidade Católica do Rio de Janeiro, 2013.

COSTA, J.; FIGUEIREDO SILVA, M. C. Nominal and verbal agreement in Portuguese. In: Studies on Agreement. Padova: Unipress, 2006. p. 25-46.

DE VOGELAER, G. et al. Bridging the gap between language acquisition and sociolinguistics. In: DE VOGELAER, G.; KATERBOW, M. (Eds.). Acquiring Sociolinguistic Variation. John Benjamins Publishing Company, 2017. p. 1-41.

EMBICK, D. The Morpheme: A Theoretical Introduction. p. 247, 2015.

EMBICK, D.; NOYER, R. Movement operations after syntax. Linguistic Inquiry, v. 32, n. 4, p. 555-595, 2001.

FERRARI NETO, J. Aquisição de número gramatical no português brasileiro: processamento de informação de interface e concordância. Tese de Doutorado. 154f. Pontíficia Universidade Católica do Rio de Janeiro, 2008.

FERRARI NETO, J.; CORRÊA, L. M. S.; AUGUSTO, M. R. A. O Processamento da Informação de Interface na Aquisição do Sistema de Número Gramatical no DP em Português Brasileiro. Anais do IV Congresso Internacional da ABRALIN. 2005. 
HALLE, M.; MARANTZ, A. Distributed Morphology and the Pieces of Inflection. The View from Building 20: Essays in Honor of Sylvain Bromberger, p. 111-176, 1993.

HENRIQUE, K. S. Variação linguística e processamento: investigando o papel da distância entre sujeito e verbo na realização da concordância verbal variável no PB. Dissertação de Mestrado. 149f. Universidade Federal de Juiz de Fora, 2016.

LABOV, W. Phonological Correlates of Social Stratification. American Anthropologist, v. 66, n. 6, p. 164-176, 1964.

LABOV, W. The Social Stratification of (r) in New York City Department Stores. In: COUPLAND N., J. A. (Ed.). Sociolinguistics. London: Macmillan Education UK, 1997. p. 168-178.

LABOV, W. Padrões sociolinguísticos (Sociolinguistic Patterns). Tradução de Marcos Bagno; Maria Marta Pereira Scherre; Caroline Rodrigues Cardoso. São Paulo: Parábola Editorial, 2008.

LEVELT, W. J. M. The Speaker as Information Processor. In: Speaking: From Intention to Articulation. Massachusetts: The MIT Press, 1989. p. 1-28.

LEVELT, W. J. M. Models of word production. Trends in Cognitive Sciences, v. 3, n. 6, p. 223-232, 1999.

LOPES, R. E. V. Estágios no processo de aquisição de número no DP do Português Brasileiro. Letras de Hoje, v. 39, n. 3, p. 157-171, 2004.

LOPES, R. E. V. Bare nouns and DP number agreement in the acquisition of Brazilian Portuguese. Selected Proceedings of the 9th Hispanic Linguistics Symposium, p. 252-262, 2006.

MAGALHÃES, T. M. V. A valoração de Traços de Concordância dentro do DP. DELTA: Documentação de Estudos em Lingüística Teórica e Aplicada, v. 20, n. 1, p. 149-170, 2004.

MARCILESE, M.; HENRIQUE, K. S.; AZALIM, C; NAME, M. C. L.; et al. Processamento da concordância variável. Revista LinguíStica / Revista do Programa de Pós-Graduação em Linguística da Universidade Federal do Rio de Janeiro, v. 11, n. 1, p. 118-134, 2015.

MARCILESE, M.; RODRIGUES, E. S.; AUGUSTO, M. R. A; HENRIQUE, K. S. Efeitos de distância linear e marcação no processamento da concordância verbal variável no PB Linear distance and markedness effects in variable subjectverb agreement processing in BP. Revista de Estudos da Linguagem, n. Thematic Issue: Experimental Linguistics, 2017. 
MILLER, K. L.; SCHMITT, C. Effects of variable input in the acquisition of plural in two dialects of Spanish. Lingua, v. 120, n. 5, p. 1178-1193, 2010.

MILLER, K. L.; SCHMITT, C. Variable Input and the Acquisition of Plural Morphology. Language Acquisition, v. 19, n. December, p. 223-261, 2012.

MILROY, J.; MILROY, L. Linguistic change, social network and speaker innovation. Journal of Linguistics, v. 21, n. 02, p. 339, 1985.

MIYAGAWA, S. Why Agree? In: Why Agree? Why Move? Unifying AgreementBased and Discourse-Configurational Languages. Cambridge, Massachusetts: The MIT Press, 2010. p. 1-29.

MOLINA, D.; MARCILESE, M.; NAME, C. Ora está, ora não está: input variável e aquisição da flexão verbal de $3^{\mathrm{a}}$ pessoa do plural no PB. Matraga, v. 24, n. 41, p. 288-309, 2017.

MUFWENE, S. S. The Ecology of Language Evolution. New York: Cambridge University Press, 2001.

MUFWENE, S. S. Language Evolution: contact, competition and change. London/ New York: Continuum International Publishing Group, 2008.

MUFWENE, S. S. Transmission, acquisition, parameter-setting, reanalysis, and language change. Bilingualism: Language and Cognition, v. 14, n. 2, p. 152-155, 2011.

NARO, A. J. The Social and Structural Dimensions of a Syntactic Change. Language, v. 57, n. 1, p. 63-98, 1981.

NARO, A. J.; SCHERRE, M. M. P. "Drifting Toward the Standard Language: A Panel Study of Number Concord in Brazilian Portuguese." In: CACOULLOS, R. T.; DION, N.; LAPIERRE, A. (Eds.). Linguistic Variation: confronting fact and theory. New York. Routledge, 2015. p. 356.

NEVINS, A.; PARROTT, J. K. Variable rules meet Impoverishment theory: Patterns of agreement leveling in English varieties. Lingua, v. 120, n. 5, p. 1135$1159,2010$.

PACE, A. et al. Identifying Pathways Between Socioeconomic Status and Language Development. Annual Review of Linguistics, v. 3, n. 1, p. 285-308, 2017.

PERKINS, S. C.; FINEGOOD, E. D.; SWAIN, J. E. Poverty and language development: roles of parenting and stress. Innovations in clinical neuroscience, v. 10, n. 4, p. 10-9, abr. 2013. 
PESETSKY, D.; TORREGO, E. "The Syntax of Valuation and the Interpretability of Features". In: KARIMI, S.; SAMIIAN, V.; WILKINS, W. (Eds.). Clausal and Phrasal Architecture: syntactic derivation and interpretation: in Honor of Joseph E. Emonds (Linguistik Aktuell / Linguistics Today). John Benjamins Publishing Company, 2007. p. 262-294.

RITTER, E. On the syntactic category of pronouns and agreement. Natural Language and Linguistic Theory, v. 13, n. 3, p. 405-443, ago. 1995.

ROELOFS, A; MEYER, A S.; LEVELT, W. J. A case for the lemma/lexeme distinction in models of speaking: comment on Caramazza and Miozzo (1997). Cognition, v. 69, n. 2, p. 219-230, 1998.

ROELOFS, A. Rightward incrementality in encoding simple phrasal forms in speech production: Verb-particle combinations. Journal of Experimental Psychology-Learning Memory \& Cognition, v. 24, n. 4, p. 904-921, 1998.

ROORYCK, J. On two types of underspecification: Towards a feature theory shared by syntax and phonology. Probus, v. 6, n. 2-3, p. 207-234, 1994.

SCHERRE, M. M. P. A regra de concordância de número no sintagma nominal em português. Dissertação de mestrado. 159f. Pontifícia Universidade Católica do Rio de Janeiro, 1978.

SCHERRE, M. M. P. A concordância de número nos predicativos e nos particípios passivos. Organon, n. 18, p. 52-70, 1991.

SCHERRE, M. M. P. Aspectos da concordância de número no português do Brasil. Revista Internacional de Língua Portuguesa (RILP) - Norma e Variação do Português, p. 37-49, 1994.

SCHERRE, M. M. P.; NARO, A. J. Sobre a concordância de número no português falado do Brasil. (G. Ruffino, Ed.) Dialettologia, geolinguistica, sociolinguistica. (Atti del XXI Congresso Internazionale di Linguistica e Filologia Romanza). Centro di Studio Filologici e Linguistici Siciliani, Universitá di Palermo. Tübingen: Max Niemeyer Verlag, 5:509-523, 1998.

SCHILLER, N. O.; COSTA, A. Different selection principles of freestanding and bound morphemes in language production. Journal of Experimental Psychology: Learning Memory and Cognition, v. 32, n. 5, p. 1201-1207, 2006.

SHI, R. Functional Morphemes and Early Language Acquisition. Child Development Perspectives, v. 8, n. 1, p. 6-11, mar. 2014.

SILVA, C. R. T.; MAGALhÃES, T. M. V. Sobre a natureza do morfema de pluralidade em português: rediscutindo o domínio da concordância. Veredas, v. 18, n. 2003, p. 102-122, 2014. 
SLOBIN, D. I.; WELSH, C. A. Elicited imitation as a research tool in developmental psycholinguistics. Report Resume. 21f. 1967.

SQUIRES, L. Social Differences in the Processing of Grammatical Variation. UPenn Working Papers in Linguistics, v. 20, n. 2, 2014.

STEMBERGER, J. P.; MACWHINNEY, B. Frequency and the lexical storage of regularly inflected forms. Memory \& cognition, v. 14, n. 1, p. 17-26, 1986.

TAFT, M.; FORSTER, K. I. Lexical storage and retrieval of prefixed wordsJournal of Verbal Learning and Verbal Behavior, 1975.

VIEIRA, S. R. Padrões de concordância de terceira pessoa plural no português: um balanço das contribuições do projeto 21 da ALFAL. Cuadernos de la ALFAL. 2015 .

VIEIRA, S. R.; BRANDÃO, S. F. Tipologia de regras linguísticas e estatuto das variedades/ línguas: a concordância em português. Lingüistica, v. 30, n. 2, p. 81112, 2014.

YANG, C. D. Knowledge and Learning in Natural Language. New York: Oxford University Press, 2002.

Ana Paula da Silva Passos Jakubów AnaPaulaSPJ@gmail.com

Letícia M. Sicuro Corrêa

LSCorrea@puc-rio.br

Recebido em: 10 mar. 2018

Aceito em: 10 jun. 2018

Publicado em: 19 ago. 2018 